\title{
Knowing by ear: leveraging human attention abilities in interaction design
}

\author{
Saskia Bakker · Elise van den Hoven • Berry Eggen
}

Received: 20 December 2010 / Accepted: 20 June 2011 / Published online: 12 July 2011

(C) The Author(s) 2011. This article is published with open access at Springerlink.com

\begin{abstract}
In a world in which intelligent technologies are integrated in everyday objects and environments, users are at risk of being overburdened with information and interaction possibilities. Calm technology therefore aims at designing interactions that may reside in the periphery of the user's attention and only shift to the center of the attention when required. However, for such designs to be effective, a detailed understanding of human attention abilities is needed. In this paper, we therefore present a qualitative study on the everyday periphery of the attention. As we expected, we found that sound plays a major role in this, which supports our approach to use interactive sonification as an interaction style for peripheral interaction. We present a range of rich examples of everyday situations that lay out the design space for peripheral interaction and support these findings by describing three initial designs that use interactive sonification for peripheral interaction.
\end{abstract}

Keywords Interaction design · Periphery · Attention · User-centered design · Audio · Interactive sonification

\section{Introduction}

The role of the computer is rapidly changing in everyday life. Computing technology is being integrated in everyday objects and environments. As a result, information can

\author{
S. Bakker $(\varangle) \cdot$ E. van den Hoven $\cdot$ B. Eggen \\ Department of Industrial Design, Eindhoven University \\ of Technology, Eindhoven, The Netherlands \\ e-mail: s.bakker@tue.nl \\ E. van den Hoven \\ e-mail: e.v.d.hoven@tue.nl \\ B. Eggen \\ e-mail: j.h.eggen@tue.nl
}

be everywhere nowadays. Interaction through or with these kinds of technologies typically happens through screens, keyboards, mouses, touch-screens and the like. However, as these types of interfaces generally require the user's focused attention, in a world of ubiquitous computing, we are at risk of being overburdened with information [1].

When looking at the physical world however, lots of information is present there too; the weather, time, mood of people around. Yet these things are usually not experienced as a burden, since they are easily ignored and only focused on when relevant. For example, when driving a car, one is generally not focused on the sounds of the engine, but will immediately notice a sound resulting from a defect. Clearly, we have developed abilities that enable us to perceive information in the periphery of our attention, while we may also focus on it in the center of our attention when needed or desired. As a result of these human attention abilities, such information streams do not overburden us at all.

Interestingly, most current methods of human computer interaction do not leverage these attention abilities. However, with the upcoming ubiquity of computing technology, it may be interesting to investigate if we can gain inspiration in the way we interact with the physical world when designing interactions with the digital. This way, interaction could shift to the periphery of the attention and only engage the center when relevant, enabling technology to seamlessly fit into our physical lives. This is highly related to Weiser and Brown's [2] vision of calm technology, "technology that engages both the center and the periphery of our attention and in fact moves back and forth between the two" [2, p. 79]. In the realm of this vision, our research aims at studying how to design interactive systems that can reside in the periphery of the attention but shift to the center when desired.

Although many current interactive systems rely on the visual modality (e.g. screens), we see a lot of potential 
in audio as a modality for interactive peripheral systems. In everyday situations, audio seems to play a major role in creating awareness of the surroundings, for example through the cocktail party effect [3]. In addition, one does not have to look at the source to perceive auditory information [4], which enables hearing it while visually focused on something else. We therefore think that interactive sonification [5], an area that uses sound during interaction with computing systems, would be an interesting interaction style for our purpose.

Several examples are known of calm technology (e.g. [2, $6,7])$, some of which use audio to present information (e.g. [8]). Though these examples provide an interesting overview of the research area as well as point out some insights for design, hardly any of these designs are based on extensive user-centered research. Since our aim is to fit technologies in everyday life by designing interactions inspired by interactions we have in the real world, we are interested in exploring how human attention abilities are used in everyday life, in order to inform the design of interactive systems that leverage these abilities. In this paper, we therefore present an extensive qualitative study on peripheral perception in everyday situations. Although we expect that audio plays a major role in this, we have studied all modalities, in order to verify (or falsify) our expectations.

\section{Sonification as calm technology}

Sound or sonification is used in several interactive systems, such as for alerts, status indications or entertainment [9]. Interactive sonifications mainly use sound to support interaction or data exploration [5]. This is useful when users need to visually focus on something else, or when immediate action is required. As a result, these sounds are mostly designed to be in the center of the listener's attention.

In line with the previously mentioned vision of calm technology [2], some areas of research do aim at employing the periphery of the user's attention. Peripheral displays "allow a person to be aware of information while she is attending to some other primary task or activity" [7, p. 247]. Motion Monitor for example uses colored light to provide awareness of movements at remote locations [7]. Similarly, Markopoulos describes awareness systems [10], which enable users to maintain awareness of activities of others.

Although these examples mainly use the visual modality to display information, some systems also use sonification as calm technology. The Dangling String [2] for example is a plastic string connected to a motor, that rotates based on the activity of the Ethernet cable in an office. This creates a characteristic noise indicating the office's network activity. Another example is Audio Aura [11], which provides office workers with relevant information via background auditory cues. AmbientROOM [8], uses a background soundscape of nature sounds to convey information about emails or the stock market. A more recent example is Birds Whispering [6], which conveys information about the activity in an office through bird sounds. Home radio [6] supports connectedness to the home while away, by making utility streams (e.g. gas, water) audible. Mauney and Walker [12] present a system that enables stock traders to monitor stock market changes while visually attending something else.

While most of these examples motivate their design choices by referring to everyday interaction or perception in the physical world, hardly any support this with extensive experiential data about such interactions. We argue however, that thorough investigation of everyday peripheral perception could significantly benefit the design of interactive systems that are to be used in such situations.

\section{Attention theory}

In the areas of psychology and neuroscience, several theories of attention have been developed. In this section, we present our current understanding of human attention abilities based on attention theory.

From the second half of the twentieth century on, many attention studies focused on the auditory modality. Research on the cocktail party effect [3] revealed that although in many situations multiple auditory channels are to be heard, subjects are easily able to focus their attention on (or select) one channel and ignore (or reject) others. This selection of auditory streams is not only influenced by choice, but also by salience [13] as well as by a cognitive process called priming [14]. A sudden loud noise for example has such salient physical properties that it is immediately selected. Furthermore, streams that contain highly relevant stimuli are more likely to be selected as a result of priming. This is commonly studied by letting subjects hear two channels of spoken information and instruct them to attend to one and reject the other. Such experiments revealed that when one's own name [15] or words similar to those in the attended channel [14] were present in the rejected channel, the subjects tended to notice them. This shows that those stimuli are primed.

Apart from attending to sensorial stimuli such as audio cues, one may also focus the attention on actions. Such actions can include sensorial, bodily and cognitive processes. Although the before described selection of sensorial stimuli plays an important role in the attention process, attention is commonly described as the division of a limited amount of mental resources over different activities [16], also referred to as divided attention theory [17]. According to this theory, the extent to which we can perform multiple activities at once depends on the mental effort required for each task. 
Fig. 1 Illustration of the center and periphery of the attention: division of resources during a high attentional task (left) and a combination of low attentional tasks (right). Vertical bars represent potential activities that are performed when resources (white circles) are allocated to them
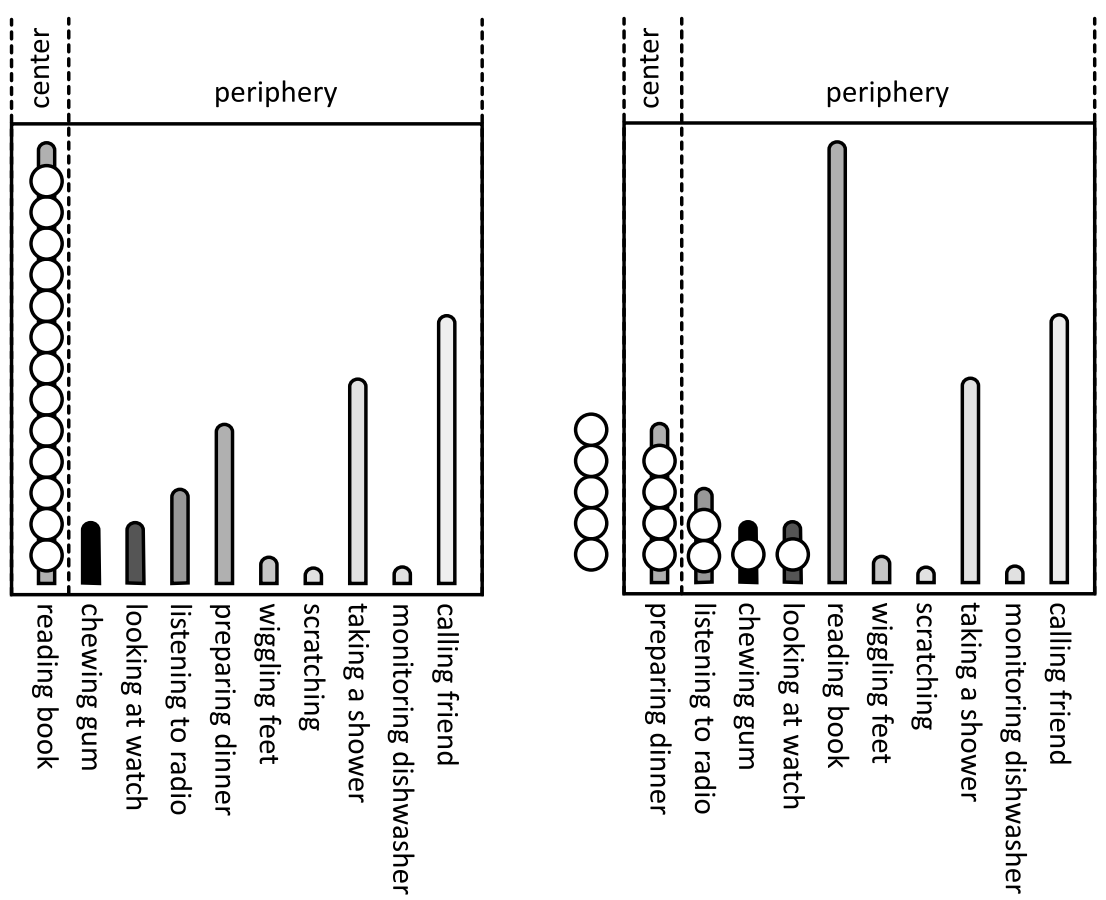

In Fig. 1, we present an illustrative overview of our understanding of attention process, which is elaborately described in [18]. Potential activities (that one can undertake but that are not necessarily being performed at the moment) are illustrated as vertical bars of which the height indicates resource demand. The brightness of the color of these bars indicates the likelihood of them being selected, based on priming and salience; the darker the bar, the more likely the activity is to be selected. Mental resources are indicated as white circles.

In psychology literature, the word periphery is generally used in the context of visual perception, indicating the parts of vision that occur outside the center of the visual field [17]. In the area of ubiquitous computing, the word periphery is used to indicate "what we are attuned to without attending to explicitly" [2, p. 79]. In line with our understanding of the attention process, we see the center of the attention as the one activity to which most resources are allocated and the periphery of the attention as all other potential activities, regardless of whether resources are allocated to them or not, see Fig. 1. Although our illustration seems static, the attention process is highly dynamic as the division of resources over activities is subject to constant change.

\section{Everyday periphery study setup}

The aim of the presented research is to study how to design interactive systems that can be monitored in the periphery of the user's attention and shift to the center when this is required. Attention theory provides an understanding of the processes that underlie the allocation of mental resources [18], but such theories are based on experiments in controlled laboratory settings (e.g. $[3,14,16])$. Though useful from a psychology point of view, these experiments have little resemblance to everyday situations, which involve several stimuli and different activities requiring diverse amounts of resources. Knowledge regarding how to design peripheral stimuli that are usable in an everyday context can therefore hardly be gathered through such controlled studies. Rather than gathering specific data to quantify a hypothesis about attention skills, we are interested in gathering rich qualitative data to inform the design of peripheral interactions.

To gain insight in how the periphery of the attention is 'used' in everyday life, we set up a qualitative user study. Although the study concerned both perceptions and actions that may take place in the periphery, this paper only focuses on the perception of everyday stimuli and how this may shift between center and periphery of the attention. This allows us to lay-out and verify the design space for systems that can be monitored in the periphery of the user's attention.

Most related work focuses on applications for offices (e.g. $[6,12])$, probably because monitoring information and supporting social cohesion play an important role here. However it also brings along limitations, such as the fact that audio should not disturb other workers. Furthermore, office workers may often be highly concentrated, which may allow less opportunities for peripheral interactions. The home environment seems a place where people are less often concentrated and where audio is less disturbing as user(s) will 
have introduced it themselves. We therefore decided to focus our study on the home environment.

As people are by definition not consciously aware of what is happening in the periphery of their attention, our goals will not be reached by directly interviewing participants about it. Observations intrude people's everyday life in such a way that observed scenarios may become unrealistic. Therefore, we think that a suitable way to reach our goals is to use the method 'context mapping' [19]. In context mapping studies, participants are 'sensitized' for the topic of interest before involving them in a group discussion. Although context mapping studies usually focus on specific design problems [19], we think that a similar approach is useful for our research because it would allow for a period of reflecting on the way participants 'use' their periphery, before we interview them about it. Furthermore, as context mapping involves different phases, the topic can be approached in multiple ways, which may increase the richness of the data.

In line with the context mapping approach [19], our study consisted of two phases; the sensitizing phase and the discussion phase. Although we expect that audio plays an important role in everyday perception, we address all senses in our study. For example, we asked "what do you perceive?" rather than "what do you hear?". In this section we will describe the study design, which was iterated once through a pilot study with two participants.

\subsection{Participants}

For this study, we have recruited 13 participants. Since we do not have a clear target group for our future designs, we selected a diverse group of participants. In this selection, we considered age (between 23 and 60, mean age 38), gender ( 6 male, 7 female) and occupation ( 3 engineers, 2 researchers, 2 teachers, 2 housewives, 2 psychologists, 1 journalist, 1 manager). Most participants were highly educated, which we saw as an advantage, as they may be used to abstract thinking, which could be useful during our study. In addition, we considered other factors that may influence people's activities at home, namely dwelling type ( 3 lived in an apartment, 2 in a row house, 3 in a semi-detached and 5 in a detached house) and family living situation (2 lived alone, 6 with their partner and 5 with partner and children). Due to external circumstances, 2 participants only participated in the sensitizing phase.

\subsection{Sensitizing phase}

The aim of the sensitizing phase was to make the participants reflect on the way they use their periphery during everyday activities at home. This phase lasted for one week. In the beginning of the week, the participants were given
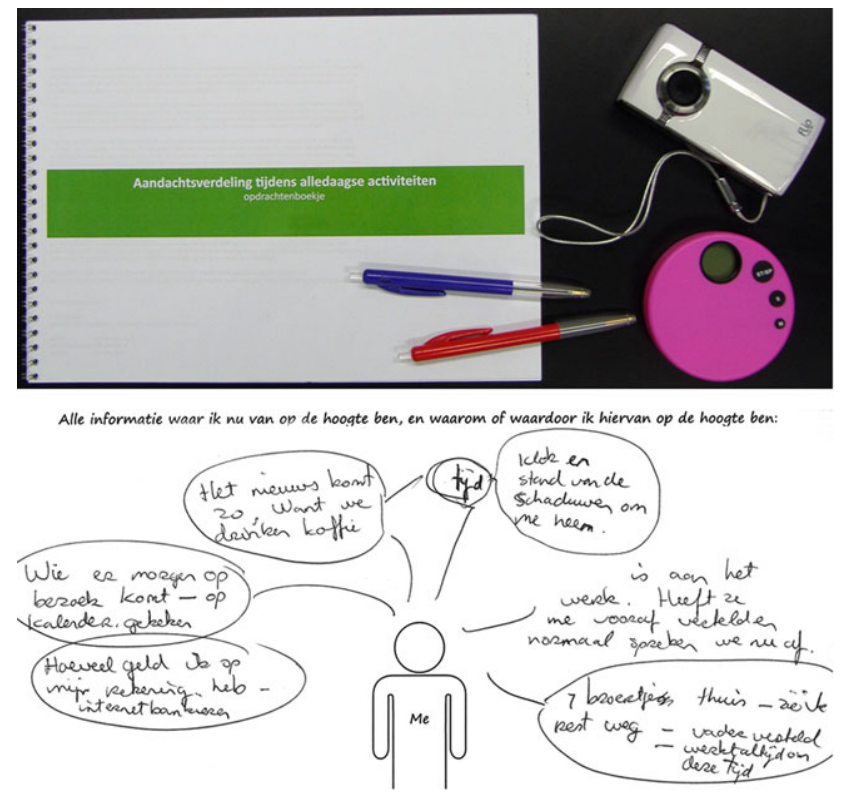

Fig. 2 The sensitizing package (top) and an example of the result of a sensitizing exercise (bottom), in Dutch

a 'sensitizing package' consisting of a booklet with six 10minute exercises and materials needed to perform them (see Fig. 2). The exercises were meant to trigger the participant to explore and think about everyday stimuli they perceive without directly paying attention to them. For example, participants were asked to consciously notice everything they can hear, see or smell in their home, or to record a video of an everyday activity and reflect on their (peripheral) behavior while looking at this video.

Since we are interested in everyday situations in the home environment, each participant was instructed to perform the exercises in his or her own home at a moment of choice during the week. The participants were not allowed to perform more than two exercises on the same day.

The sensitizing phase is highly similar to the approach of cultural probes [20], a method used to gather inspirational data from users. Although we also used the results of the sensitizing exercises in the analysis of our study, their main goal was to prepare participants for the discussion phase.

\subsection{Discussion phase}

After having performed the sensitizing exercises at home, the participants were invited for a creative interview session in groups of three or four. Although the sensitizing phase was individual, sharing experiences was expected to stimulate discussion and result in richer data. The sessions, which were captured on video, lasted about 90 minutes, were led by a researcher and consisted of three separate parts; a group interview and two exercises.

The first part of the discussion phase was a group interview consisting of open questions to gather everyday experi- 


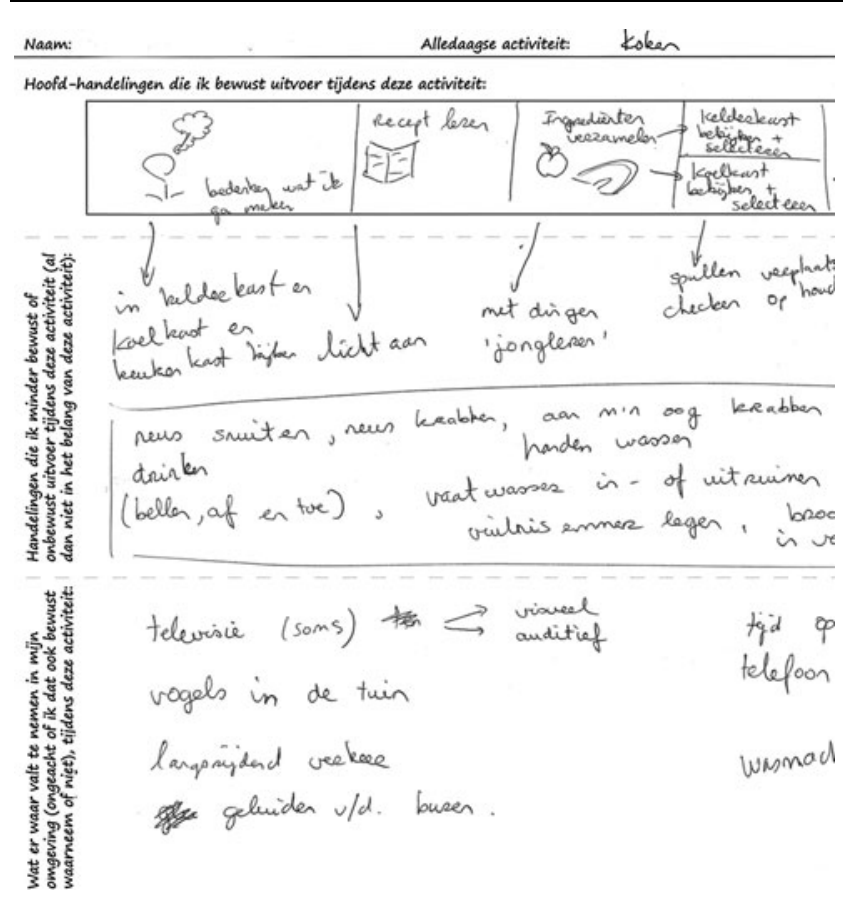

Fig. 3 Example result of the scenario exercise, in Dutch

ences regarding peripheral perception. To ensure that participants discussed the periphery of their attention rather than the center, we mainly asked what they perceived apart from their main focus of attention. For example "when you are doing everyday activities at home, what can you perceive that is not related to your activity?".

After the group interview, the participants were given an exercise, named the 'scenario exercise'. This exercise was meant to obtain specific examples of peripheral perception. We asked the participants to describe an everyday activity of their choice, for example cooking or having breakfast. The participants first created a timeline describing the main actions they perform during this activity. In addition, the participants were asked to write down what they perceive during this activity. This may be linked to the timeline (e.g. 'when laying the table, I hear my husband taking a shower'), or not (e.g. 'while cooking, I usually see children playing outside'). See Fig. 3 for an example of a scenario. After finishing the scenarios, they were discussed in the group.

The final exercise of the discussion phase was aimed at gathering inspiration for the design of systems that be monitored in the periphery of the attention. The main objective was to find out what kind of information people would want to be aware of through such systems. For this exercise, we anticipated that the participants had gained an understanding of the fact that they are constantly aware of all kinds of information (e.g. the weather, the time) without experiencing this as a burden. We therefore expected that the participants could be able to think about what other information they would like to be aware of in a similar way. To emphasize

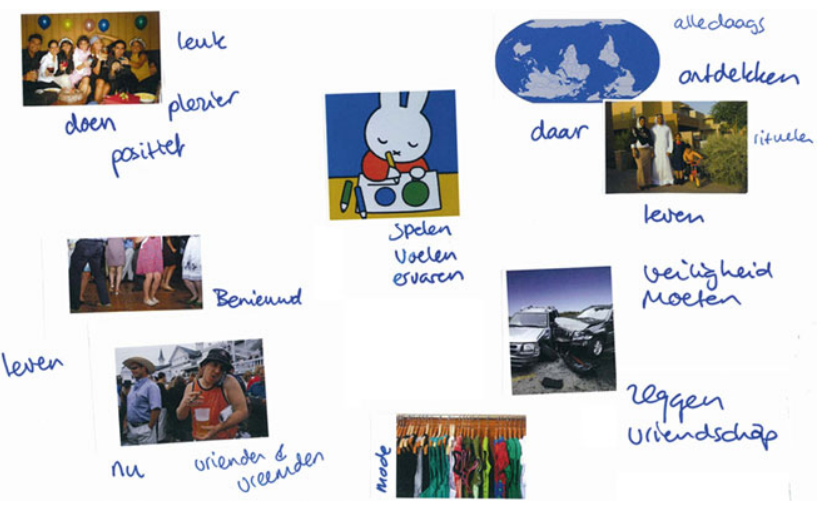

Fig. 4 Example result of the collage exercise, in Dutch

that the ideas of the participants would not have to be realistic, we formulated the exercise as follows: "if you would have a 'sixth sense', that would enable you to always be aware of certain information of your choice, which kind of information would you want to be aware of?". To give the participants, who may not be experienced in brainstorming, a starting point, we provided them with 111 small images of diverse situations and things, as well as 102 words that could inspire them. With these materials, we asked the participants to create a collage that explains what they would want to put in their 'sixth sense', see Fig. 4.

\subsection{Data analysis procedure}

Having performed both the sensitizing and the discussion phase with our participants, we had gathered several types of qualitative data. In the analysis of this data, we were mainly interested in gaining insight in how the periphery is 'used' in everyday life to inform our future designs. Although we naturally had expectations of how the everyday periphery is used, we did not have a specific hypothesis to be tested. We therefore analyzed the data using 'open coding' [21] or 'conventional content analysis' [22], which is "used with a study design whose aim is to describe a phenomenon (..), when existing theory or research literature on the phenomenon is limited" [22, p. 1279]. This method entails that data is clustered without a predefined coding scheme. Instead, detailed clusters emerge during the analysis.

To enable analysis, the videos taken during the discussion sessions as well as the handwritten results of the sensitizing and discussion exercises were transcribed verbatim. From these transcriptions, quotes were selected that seemed to capture examples or insights related to perception that may shift between periphery and center of the attention. This led to a selection of 277 quotes, which could be short statements (e.g. "I hear music while cooking") or richer explanations (e.g. "I am sitting on the couch reading, but I am actually occupied with "where are my children playing?"). 
In the creative collage exercise, the participants did not describe examples of peripheral perception. We therefore gathered no quotes from this exercise. The collages have been used as inspiration for the design process and will be discussed in the section "Peripheral Interaction Concepts".

To find common themes in these quotes, they were used as input for a group clustering session, in which three researchers participated, two of whom had not been involved in the data gathering. The 277 quotes were printed on small pieces of paper and divided among the researchers. Since the aim of our study is to inform the design of interactive systems that can engage the periphery of the user's attention and shift to the center when required, we were most interested in gaining insights in the preconditions that enable peripheral perceptions or shifts between periphery and center of the attention. We therefore asked the researchers to cluster their quotes in a way that it would address the question 'why is a stimulus (not) perceived?'. The researchers first individually made a clustering before they compared their clusters. After discussing the differences, the three researchers agreed to an overview of clusters that captured the essence of the data.

As a result of the group clustering session, not only clusters describing different preconditions for peripheral perception were found, we also concluded that an overview of the types of perceptions (e.g. hearing, seeing) would be useful for the analysis of the data. This could provide a direction for peripheral interactions. We therefore decided that to provide a valuable overview of the data, the quotes should be grouped on a two-dimensional scale; one dimension indicating why stimuli are (not) perceived and one dimension indicating the type of perception. To finalize the data analysis, two researchers acted as coders who independently clustered the quotes according to these two dimensions. These coders were the first author and a researcher who had not participated in the clustering session. After both coders had clustered all quotes, the divisions of quotes were compared and discussed. As a result, some quotes were moved between clusters to correct mistakes.

Comparison of the two divisions of quotes enabled an evaluation of the reliability of the clusters. This was done using Cohen's Kappa statistic, which provides the agreement between two coders as a number between 0 and 1 ( 0 indicating no agreement and 1 indicating perfect agreement) [23]. As a result, we found a Kappa Coefficient of 0.88, which is generally regarded as high agreement [24].

\section{Findings}

The objective of our study is to gain insight in the way the periphery of people's attention is used in the everyday home environment, particularly concerning the perception of sensorial stimuli. A detailed understanding of this phenomenon is needed to inform the design of systems that leverage human attention abilities in interaction with technology. To gain this understanding, 277 quotes selected from several parts of the study, have been clustered to find common themes regarding why certain perceptions can (not) take place in the periphery of the attention. In this section, we will discuss the results of this clustering.

\subsection{Why everyday stimuli are (not) perceived}

As a result of the group clustering session, we identified four main clusters that each describe a factor that may influence the perception of stimuli and therefore facilitate shifts between the periphery and center of the attention. These four main clusters (content of the stimulus, expectations of the perceiver, current activity of the perceiver and other factors) as well as the sub-clusters that were identified, will be discussed here in detail.

Content of the stimulus The first factor that seems to influence the perception of stimuli is the content or meaning of the stimulus. In this cluster, we found most quotes (65 out of 277).

In the scenario exercises performed during the discussion phase, the participants were asked to describe an everyday activity they perform at home in detail. One participant described how she cleans her house. While writing down the steps she takes when cleaning, she mentioned at one point, "it suddenly comes to my mind that while cleaning, I always notice more things that have to be cleaned, that I do not notice otherwise. For example when sweeping the floor, I see some dirt on the window, which must have been there for days, but I have not seen it before". Similarly, another participant described in a sensitizing exercise "When doing the laundry, I suddenly notice a sock lying in my child's room, that also needs to be washed. I would not have noticed this otherwise". These examples indicate that stimuli related to your current activity may attract your attention. The same seems to hold for stimuli that relate to a future activity. For example, when asked what she perceives during breakfast, a participant said "I notice that the peanut butter is almost empty, so I have to buy a new jar when I am in the store".

When asked to name examples of things she perceived during everyday activities, a participant mentioned that while cooking, her attention often wandered off to things happening in the street that she can see through the window, particularly when children were playing there. This attracted her attention because she enjoyed watching their fun and it reminded her of the time her children were younger. This example may indicate that a stimulus which evokes certain emotions, in this case positive emotions, easily attracts the attention. This also seems to hold for stimuli that evoke negative emotions. For example, another participant noted that when at home, he always knows if his upstairs neighbor is 
home. He experiences this person as 'annoying' and mentioned that this made him notice almost every sound.

Furthermore, we saw that if the content or meaning of the stimulus is personally relevant, it is also easily noticed. For example, one participant mentioned that she is always aware of what her cat is doing, even if it is not attracting attention. When discussing what causes this awareness, she came to the conclusion that she feels the cat is part of the family and it is therefore important for her to monitor its activity. Another participant mentioned "I am allergic to mess, so whatever I am doing or wherever I am in my house, I always see mess". Another participant reacted to this by saying "I don't experience this at all, my house is often messy, but it does not bother me". Apparently, certain kinds of information (e.g. activity of the cat or the cleanness of the house) are personally highly relevant to some participants and related stimuli are therefore quickly noticed.

Expectations of the perceiver Another factor that seemed to influence the perception of stimuli is the expectations of the perceiver. In this cluster, we found 36 out of 277 quotes.

In one of the discussion sessions, a participant addressed an exercise he had performed in the sensitizing phase. In this exercise, he had to write down everything he was aware of at that moment. One of the things he wrote down was "I thought that the cat was outside, but I hear it moving on the kitchen table, so it is indoors after all". In another discussion session, a participant described a situation in which he had turned on the dishwasher about 15 minutes ago, "while I was already doing something else, I noticed that I did not hear any sounds from the dishwasher. I looked and it was not on". In both these examples, the participant had a certain expectation before hearing a sound (or in the second example the absence of a sound) that was not in line with his expectation. The first participant may not have noticed the cat's sound if he expected that it was indoors, and the second participant would likely not have noticed the absence of the dishwasher sound if he did not expect the machine to be on. The sounds would then be in line with their expectations. This was also seen in other discussions. For example another participant, who lived near a busy road, mentioned that although rather loud traffic sounds are to be heard in his apartment, he normally does not notice them at all. However, he remembered an incidence where the road was blocked and no traffic was to be heard. Interestingly, he immediately noticed the absence of the (expected) sound.

Current activity of the perceiver Resulting from our data, we furthermore found that it may depend on the current activity of the perceiver if a stimulus is perceived or not. 32 quotes were identified in this cluster.

Several participants indicated that when their current activity requires a lot of attention, e.g. when reading a book, they do not notice stimuli that they would notice otherwise. Even when someone directly speaks to them, they may not notice it. For example "sometimes when I am reading, someone calls me or asks a question, but this does not reach me, so I do not answer". On the other hand, we also saw that when little or no attention is paid to an activity, external stimuli are quickly noticed. For example "when I am watching TV, but nothing interesting is on, then I notice everything that happens around me". In few cases, we saw that the perceiver made a choice not to pay attention to a stimulus because this would interfere with his current activity. For example "If I am watching an exciting movie, and someone asks me something, then I do not answer, otherwise I will miss something".

Other factors Apart from the previously mentioned clusters, we found 25 quotes indicating other factors that can influence everyday perception. These quotes showed that the perception of stimuli may be influenced by physical properties of the stimuli (e.g. "a very loud sound attracts the attention"), personal factors of the perceiver (e.g. "I personally do not smell very well, so I often do not notice strange smells"), or environmental factors (e.g. "only when it is very quiet, I can hear trains in my house").

Apart from the factors mentioned above, we also gathered a large number of quotes for which it was unclear why the stimulus was (not) perceived. For example "while cooking, I perceive the washing machine". This could relate to a future activity, be unexpected or be perceived as annoying. These 119 quotes were clustered as 'unclear'.

\subsection{Types of peripheral perception}

Apart from the overview of factors that may influence the perception of everyday stimuli, the quotes were also clustered on a second dimension, indicating the type of perception (hearing, seeing, smelling, tasting, touching or unclear). The overall overview of the number of quotes in each cluster of these two dimensions is provided in Table 1.

When looking at the different types of perception shown in Table 1, we clearly see a higher number quotes related to hearing (125 out of 277 quotes) compared to other modalities. This is a noticeable result, since all our questions related to perception were deliberately formulated in such a way that no preferred modality was indicated (e.g. 'what do you perceive?' rather than 'what do you hear?').

\section{Discussion}

The aim of the study described in this paper is to gain an understanding of how the periphery of the attention is used in everyday situations. This information is needed to inform 
Table 1 For each (sub-)cluster indicating why a stimulus was perceived, the number of quotes assigned by the first author, categorized over the different possible types of perception. For example, the first coder found 7 quotes that indicated auditory stimuli that were perceived because the content of these stimuli related to the current or future activity of the perceiver

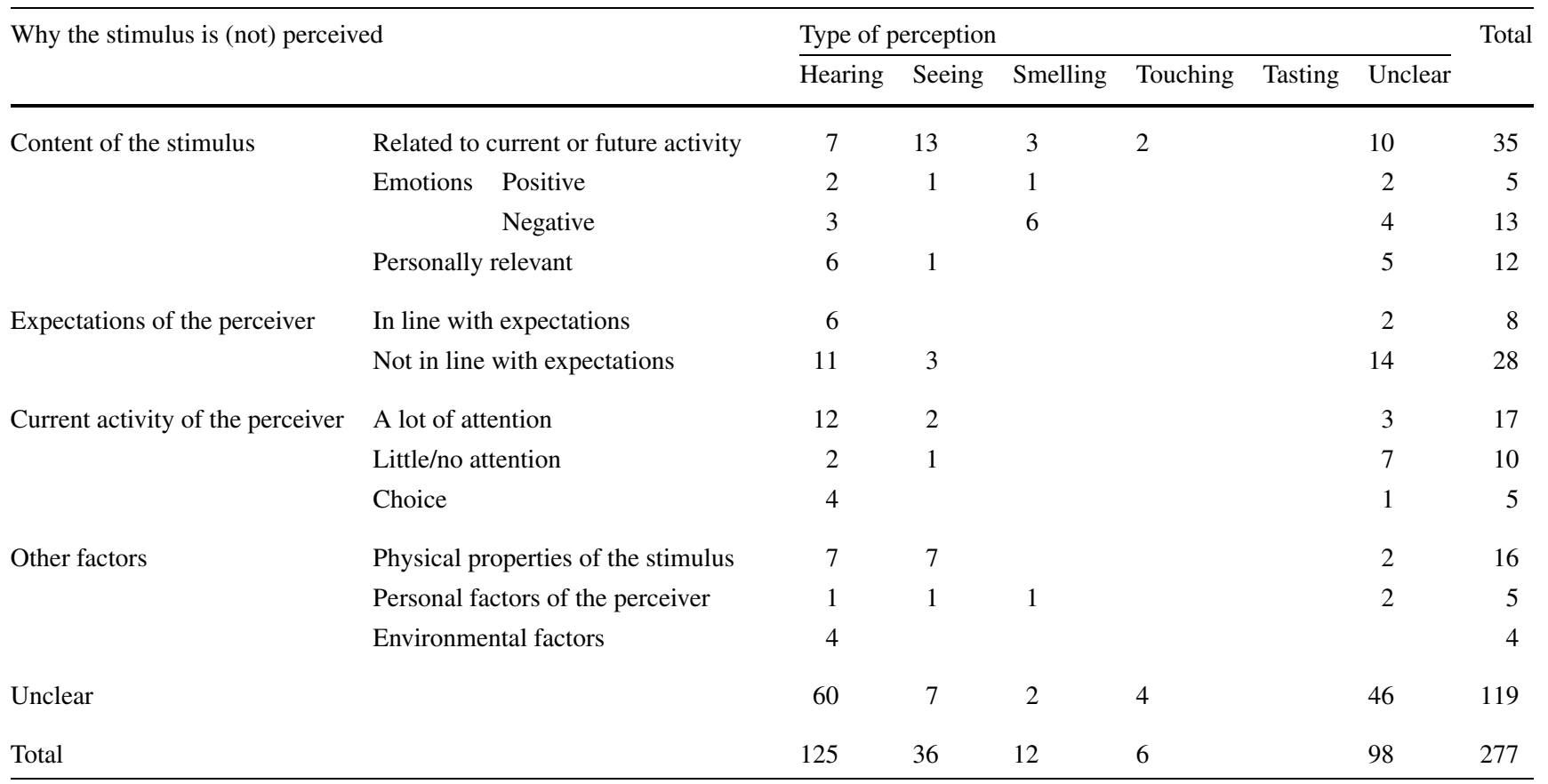

the design of interactive systems that can reside in the periphery of the attention and shift to the center when desired. In this section, we will discuss our current understanding of the everyday periphery of the attention. Furthermore, we will discuss the design implications of our results. To support our findings however, we will first address the methodology we used in this study.

\subsection{Methodology}

In our study, we were interested in finding a diverse range of qualitative examples of how the periphery of the attention is used during everyday activities in the home environment. Based on attention theory [16, 17], we have described the periphery of the attention as all potential activities that one can undertake at a certain moment, accept the one to which most mental resources are currently allocated (see Fig. 1). Given this description, which entails that little or no mental resources are allocated to peripheral activities, one may question if it is at all possible to interview people about the periphery of their attention. We tried to overcome this problem by including a sensitizing phase in our research, to enable the participants to start thinking about and reflecting upon their use of the periphery, before the actual interview took place. During particularly the discussion phase of our study, participants frequently made remarks such as "when making the exercises at home, I started to realize how much sound is always around me", or "the exercises made me notice that I am always aware of what kind of weather it is". Such remarks gave us a strong impression that the sensitizing phase had effectively helped the participants in reflecting about the periphery of their attention, which enabled them to better discuss it in the discussion session.

However, we must also conclude that not all examples we found are clearly peripheral according to our description. For example, while cooking a participant's attention was attracted to children playing outside. This means that though cooking was in the center of her attention at first, the activity of looking outside shifted to the center and was therefore no longer peripheral. The main goal of our study is to inform the design of systems that can reside in the periphery of the attention but shift to the center when relevant for or desired by the user. We were therefore interested in all stimuli that can be in the periphery of the attention (e.g. sound or movement of children playing outside while cooking), even though they may also be focused on in the center (when paying more attention to these children than to cooking).

Furthermore, one may question if the found examples are not too influenced by the phrasing of our questions. For example the case in which a participant noticed that his cat was indoors while he expected it to be outdoors. If we would not have asked this participant "what do you perceive at this moment?', he may not have noticed the cat at all. However, it is important to distinguish the sensitizing phase from the discussion phase. In the sensitizing phase, such questions were 
asked to enable participants to become more aware of the periphery of their attention. In the discussion phase however, we only asked participants to recall experiences from their memory without focussing on the current situation. To gain a more elaborate overview of examples however, we did use the sensitizing results in the analysis, which may have influenced the overview provided in Table 1 . After looking at the data more specifically though, we found that only 62 of the 277 quotes were gathered from the sensitizing exercises, 48 of which did not clearly describe why the stimulus was (not) perceived and therefore ended up in the cluster 'unclear'. We are therefore confident that our results provide a valid overview of examples of the everyday periphery of the attention.

Table 1 shows that for quite a large number of quotes it was unclear why the stimulus was perceived. One may question if the overview of identified clusters is complete, as more or other motivations for (not) perceiving stimuli could be present in the 'unclear' cluster. However, both the first and second coder agreed that they only used the 'unclear' cluster when indeed no clear reason for perception was described. Both coders were therefore confident that no other factor is hidden in the 'unclear' cluster. The large number of quotes in this cluster could be a result of our questions 'what do you perceive at this moment' or 'what do you perceive during everyday activities?'. Even though we asked them to write down why they thought they perceived it, not all participants did write this down.

\subsection{Audio and the everyday periphery of the attention}

To obtain a better understanding of the everyday periphery of the attention, we analyzed the qualitative data gathered in our study through conventional content analysis [22].

As a result of our study, which included questions such as 'what do you perceive other than the current focus of your attention?', we found several examples of auditory perception. Some of these examples describe auditory stimuli that attract the attention (e.g. "I thought the cat was outside, but I hear it moving in the kitchen now"), while others explain how certain sounds are easily ignored (e.g. "I am used to hearing cars outside, so I don't notice them anymore"). This indicates that many sounds that are monitored in the periphery of the attention may shift to the center when they are relevant to the perceiver. Since we found many more examples of hearing compared to other modalities, see Table 1, our data confirms that audio plays a major role in the everyday periphery of the attention.

This result is remarkable, since we deliberately phrased our questions in such a way that no preferred modality was indicated. The sensitizing exercises contained questions such as 'what do you perceive at this moment?', or 'what can you perceive in your living room?'. In response, participants mostly wrote down things of a dynamic nature, for example "I hear birds outside" or "I hear my wife in the other room". Static things such as "I see a chair" or "I hear the lights humming" were hardly mentioned. These latter examples may be so obvious that the participants may not have thought about it when doing the exercises, or they may have assumed that we would not be interested in these kinds of things since we could easily conclude them ourselves. As in everyday situations, auditory stimuli are more dynamic than visual stimuli, this may have caused the large number of examples of hearing. A further explanation could be the fact that audio can be heard over a large distance; the things that you can see from your own home may be much less unexpected or new than the things you can hear. We found quite some examples of distant hearing (e.g. "I hear the neighbors", "I hear trains", "I hear there is an event at the marketsquare"), but only few such examples of seeing (e.g. "I see a man in the street that does not live here"). Although such examples were mainly found in the sensitizing phase, this has likely also influenced the discussion phase. If participants thought more about audio in the sensitizing exercise, they likely also thought more about audio during the discussions. Although these factors may have influenced the numbers presented in Table 1, the fact that we found more than twice as many quotes related to hearing compared to all other modalities together, gives us reason to believe that audio indeed plays an important role in the everyday periphery of the attention.

\subsection{Cognitive mechanisms and the everyday periphery}

Apart from indicating types of peripheral perceptions, our findings provide an overview of reasons why stimuli may (not) shift from the periphery to the center of the attention. This may for example be due to the content of the stimulus, or the expectations of the perceiver. Attention theory (e.g. [3, 13, 14]) also describes reasons for stimuli shifting from the periphery to the center of the attention; salience (e.g. a sudden loud noise) and priming (e.g. hearing your own name in an unattended conversation). When looking at our results, we see that only the cluster 'physical properties of the stimulus' describes examples of salience. Priming is a cognitive mechanism that increases the likelihood that relevant stimuli are perceived [14], e.g. those related to the current focus of attention or those that are always relevant such as your own name. Examples of priming are found in the clusters 'expectations of the perceiver' and 'content of the stimulus'. Although salient stimuli may seem most obvious to attract the attention, the cluster 'physical properties of the stimulus', contains only 16 quotes. Even if not all examples in the clusters 'expectations of the perceiver' and 'content of the stimulus' may be evident examples of priming, these clusters together contain 101 quotes. Interestingly, we must therefore conclude that priming seems to play a more important role in everyday attention than salience. 
In psychology literature, experiments on priming usually study the auditory modality and focus on speech perception $[3,14,15]$. For example; two auditory channels are presented and the subject is instructed to focus the attention on one channel, while the name of the subject is presented in the other channel [15]. Such experiments prove that one's own name is a primed stimulus. Most examples of primed auditory stimuli we found in our study however were nonspeech sounds. For example "I hear the cat in the kitchen" or "I hear my annoying neighbor". It seems as though not only words can be primed, but also other kinds of sounds.

When looking at the examples of priming found in our study, we see that these stimuli may have been primed because of expectations of the perceiver, because of an emotional content of the stimuli, because of personal relevance or because they relate to a current or future activity of the perceiver. This overlaps with theory, describing one's name [15] (personally important) as well as words related to the current focus of the attention [14] (current activity) as primed stimuli. Our results however, give the impression that apart from these two types of stimuli, other things may also be 'on the back of one's mind', that could be likely to be noticed in unattended channels (i.e. stimuli about which one has a certain expectation or those with emotional content). The idea that things 'on the back of one's mind' are easily noticed was also seen by nineteenth century psychologist James [25], who stated "If I have received an insult, I may not be actively thinking of it all the time, yet the thought of it is in such a state of heightened irritability, that the place where I received it or the man who inflicted it cannot be mentioned in my hearing without my attention bounding, as it were, in that direction, as the imagination of the whole transaction revives" [25, p. 449]. Our results provide support for this view as well as a more specified overview of the kinds of stimuli that may be primed.

Our overview of primed stimuli indicates that things 'on the back of one's mind' are likely to be noticed. Since the things that are on back of one user's mind, which depend on expectations, emotions, personal importance and current activity, will probably be different from those on the back of another user's mind, it seems as though the everyday periphery of the attention cannot easily be generalized for a large group of people.

\subsection{Designing for the everyday periphery}

The study described in this paper was aimed at gaining better understanding of the everyday periphery of the attention in order to inform the design of interactive systems that leverage human attention skills. Now that we have presented our findings, we will discuss what implications these findings have for the design of peripheral interactions.
As we have seen, hearing plays an important role in peripheral perception. As our study aims at informing the design of systems that engage the periphery of the attention, we argue that interactive sonification is a valuable interaction style for our purpose. This entails that information or feedback should best be provided through audio. Regarding the design of such peripheral auditory stimuli, the main objective should be to design audio that can be monitored in the periphery of the attention and shift to the center of the attention only when this is required, e.g. as it becomes relevant to the user. As we saw in our study as well as in literature, this can be achieved through salience or priming. Our data however showed that priming is more common in everyday situations than salience. It would therefore be interesting to see if we can leverage the process of priming in the design of interactive systems.

Several examples of calm technology systems are known that use audio to display information in the periphery of the attention. Our data provides support for this approach. However, hardly any related work is grounded in extensive usercentered research or in attention theory. An exception is research by Matthews et al. [7], which describes a "toolkit for managing user attention in peripheral displays" [7, p. 247]. Though mainly focused on visual displays, Matthews et al.'s research distinguishes different notification levels such as 'make aware' or 'interrupt'. This enables designers to assign higher notification levels to more urgent or important information. Notifications of such information will then be presented more saliently. This however, assumes that the designer can decide which information is relevant and at which moments it is relevant, which seems rather difficult. Given our results, it would entail that the system should have an understanding of the users expectation, his or her current activity, the things that are of personal relevance to the user, etc. Our results therefore suggest that presenting information multiple times in a non-salient manner should enable the user to pick up the information when it is relevant for him or her, while it can be in the periphery of the attention, otherwise. When in the periphery, the audio does not attract the attention and is thus easily ignored.

This approach would obviously not be suitable for urgent information, but seems interesting for less urgent, but still potentially interesting or relevant information. For example when a system provides auditory information that can be heard by multiple people; in case a stimulus is relevant for one user, but not for another, it should only shift to the center of the attention of the first user. This will obviously not be achieved if the stimulus gets more salient (e.g. by increasing the volume) to attract the attention. Furthermore, no intelligent system is required to decide whether the information is relevant for the user or if the user is available. Assuming that the user can easily understand the information and that it is potentially relevant, these decisions will be made by the user's peripheral cognitive processes. 
We therefore argue that auditory information should not be made louder or otherwise more distinctive when it becomes more relevant to the user, as is done now in many systems such as most computer applications that use sound. Instead, audio could be provided in a way that the user can easily pick up the presented information. When this information is relevant to the user (for example because it differs from his expectations or evokes emotions) it will likely shift to the center of the attention as a result of priming.

\section{Peripheral interaction concepts}

As a result of our study, we have obtained an understanding of the everyday periphery of the attention, as well as discussed opportunities for the design of peripheral interaction. To gain inspiration for the type of applications that may be suitable as peripheral interactions, we included an exercise in the discussion phase of our study, in which participants created collages illustrating the information that they would want to be aware of through an imaginary 'sixth sense'. Inspired by the collage results, we now present three preliminary concepts of peripheral interaction. These are meant to illustrate the design implications of our findings, as well as to provide examples of how we think the process of priming could be leveraged in interactive systems.

\subsection{Awearness}

A topic that was named by several participants in their collages was planning. For example one participant mentioned that she uses both a digital and a paper agenda. Although she hardly ever forgets appointments, a better awareness of her daily schedule would help her to prepare herself better. This could be supported by an interactive bracelet that enables such awareness through peripheral audio, see Fig. 5 . This device is wirelessly connected to your digital agenda and can provide a short sonic representation of a timeline in your agenda, for example between now and four hours in the future. This representation could be played every 15 minutes, or when requested by the user when rotating the device. In that case, the virtual location of the agenda items on

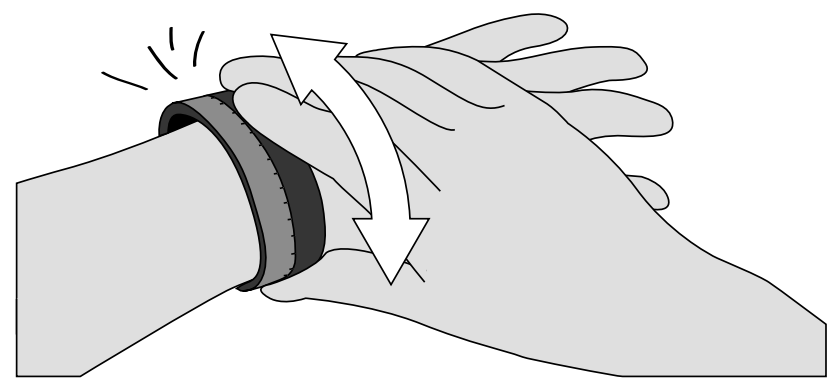

Fig. 5 Illustration of 'awearness' the bracelet indicates the time at which the item is due. In the sonic representation, different agenda items can be linked to different audio cues, which could indicate type of agenda item (e.g. group meeting, presentation, private appointment, etc.) as well as the duration or location of the meeting. An experienced user, who likely also has some knowledge about his or her agenda, is expected to be able to distinguish the different items easily. Since the information is likely personally relevant to the user, it may shift to the center at certain moments, for example when it is not in line with the expectations of the user or when it indicates that the user needs to change his current or future activity. However, since the sound will not have a relevant meaning to others, the information will remain private and may not even be noticed by others.

\subsection{Informing refrigerator}

In the discussion phase, several participants mentioned that when they open the refrigerator, for example while cooking, they usually quickly look at the other products in the refrigerator. This is done to check the expiry dates or to check for items that should be on the shopping list. Inspired by this habit, we thought of an informing refrigerator that provides a subtle auditory cue indicating which products are in the refrigerator as well as their expiry date whenever it is opened. This information could clearly be relevant for the user at the moment of opening the refrigerator, as it may relate to a current (cooking) or future (shopping) activity. The information could be provided by using a different timbre for different types of products (e.g. milk, meat, vegetables). Duration of sound in each timbre could indicate the number of products of this type and pitch could be used to represent expiry date. Although all information is presented each time the refrigerator is opened, once users have become familiar with the sonification, the sounds should easily shift to the periphery of their attention, while particularly the information that is relevant to the user (e.g. an expired milk product) will likely shift to the center of the attention. Given that users already have some knowledge of the contents of their refrigerator, they will immediately know which product of a certain type is expired.

\subsection{Connecting strangers}

In our collage exercise, four participants independently mentioned that they were interested in being more aware of activities of people whom they do not know. A participant said "If I am at a crossroad, I am busy with where I came from or where I am going. However many people stand at the same crossroad with a totally different state of mind. If I would have an impression of their thoughts, feelings or just where they came from or their destination, this would 


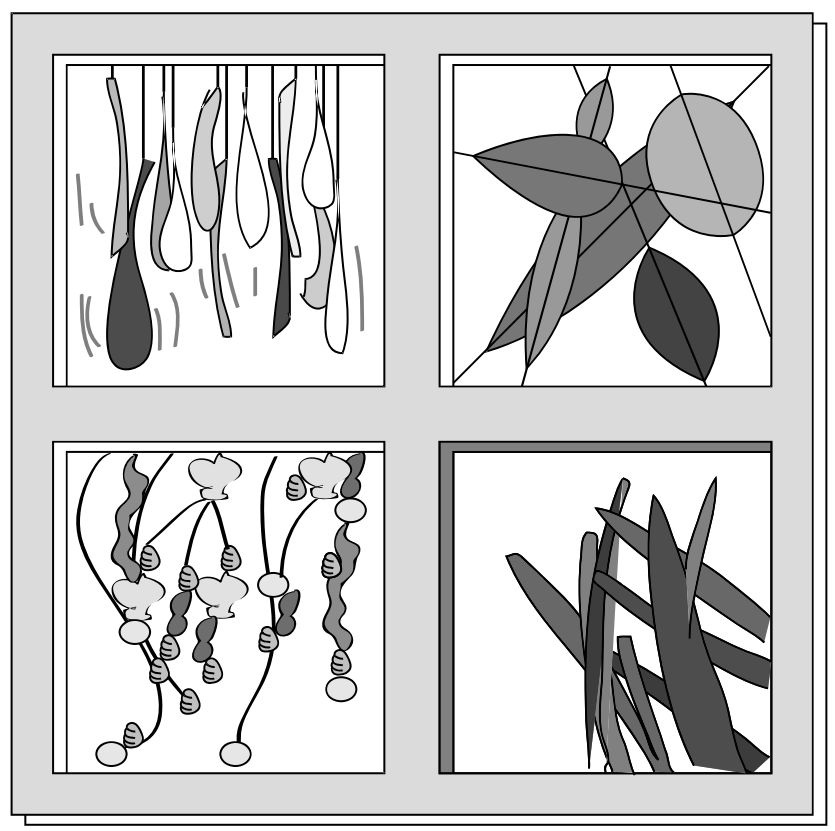

Fig. 6 Illustration of 'connecting strangers'

give me a means to put my own state of mind into perspective". Discussions on this topic revealed that the participants felt that such simple and non-newsworthy information about random strangers would help them put their situation into perspective. According to them, this could also help getting a better understanding of cultural and personal differences.

In line with this finding, we thought of a decorative information system, see Fig. 6. The installation consists of four frames of wind-chime like decorations as well as a set of sensors that register information such as movement, light intensity and temperature in the home of the user. Each frame is connected to a similar system located in an unfamiliar home. The decorations could be created by the people in the remote home to give an impression of their identity. The sensor data in the remote home is displayed in the corresponding frame as wind, light and movability of the decorations, by using actuators such as (servo-) motors and lights. This provides an audiovisual display of movement and background sounds that gives an impression of the situation at the remote location. Not knowing who they are connected to and having only little and multi-interpretable information, users will be encouraged to start thinking about who they are connected to, as well as their habits, routines and motivations. For example, when one frame is always active early in the morning, users may fantasize the remote home to be one of a baker who goes to work early, or one where a new-born baby lives who needs feeding at night, or a home in a different time-zone, etc. As a result, users may develop expectations regarding the state of the system, which will enable the information to shift to the center of the attention when it is not in line with these expectations or when positive emotions are evoked which may result from a feeling of connectedness to the remote homes.

\section{Conclusions}

In this paper, we have described a qualitative study aimed at investigating how the periphery of the attention is used during everyday activities in the home environment. This knowledge was gathered to inform the design of systems that a user can monitor in the periphery of the attention, while it may also shift to the center of the attention when this is relevant for or desired by the user.

As a result of this study, we conclude that audio plays a major role in peripheral perception. Our data therefore indicate that interactive sonification [5] seems a suitable interaction style for our purpose. In addition, we have identified specific factors that enable perceptions to shift between the center and periphery of the attention. These factors are conform to our theoretical understanding of the attention process, indicating that salience and priming can cause stimuli to shift to the center of the attention. Interestingly, we found that priming plays a more important role in everyday life compared to salience. This indicates that when leveraging human attention abilities in interaction design, priming could be an interesting cognitive mechanism to consider. We therefore argue that (non-urgent) auditory information should not be presented more saliently when it becomes more relevant, but when designed in such a way that users can easily understand the information, it should shift to the center of the attention whenever relevant to the user as a result of priming.

This work contributes to other research in the area of interactive sonification as well as ubiquitous computing in general, by qualitatively laying out a design space for an emerging type of interaction, one that can reside in the periphery of the user's attention, but shift to the center when relevant for or desired by the user.

Acknowledgements We thank the participants for investing their time in performing our (pilot-) exercises and taking part in sessions. We also thank the coders for their help in clustering and discussing the results.

Open Access This article is distributed under the terms of the Creative Commons Attribution Noncommercial License which permits any noncommercial use, distribution, and reproduction in any medium, provided the original author(s) and source are credited.

\section{References}

1. Weiser M (1999) The computer for the 21st century. Mob Comput Commun Rev 3:3-11

2. Weiser M, Brown JS (1997) The coming age of calm technology. In: Denning PJ, Metcalfe RM (eds) Beyond calculation: the next fifty years of computing. Springer, New York, pp 75-85 
3. Cherry EC (1993) Some experiments on the recognition of speech, with one and with two ears. J Acoust Soc Am 25:975-979

4. Gaver WW (1993) What in the world do we hear? An ecological approach to auditory event perception. Ecol Psychol 5:1-29

5. Hermann T, Hunt A (2005) An introduction to interactive sonification. IEEE Multimed 12(2):20-24

6. Eggen B, van Mensvoort K (2009) Making sense of what is going on 'around': designing environmental awareness information displays. In: Markopoulos P, de Ruyter B, Mackay W (eds) Awareness systems: advances in theory, methodology and design. Springer, London, pp 99-124

7. Matthews T, Dey AK, Mankoff J, Carter S, Rattenbury T (2004) A toolkit for managing user attention in peripheral displays. In: UIST'04: proceedings of the 17th annual ACM symposium on user interface software and technology. ACM, New York, pp $247-$ 256

8. Ishii H, Wisneski C, Brave S, Dahley A, Gorbet M, Ullmer B, Yarin P (1998) AmbientROOM: integrating ambient media with architectural space. In: CHI'98: conference summary on human factors in computing systems. ACM, New York, pp 173-174

9. Walker BN, Nees MA (in press) Theory of sonification. In: Hermann T, Hunt A, Neuhoff J (eds) Handbook of sonification. Academic Press, New York. http://sonify.psych.gatech.edu

10. Markopoulos P (2009) A design framework for awareness systems. In: Markopoulos P, de Ruyter B, Mackay W (eds) Awareness systems: advances in theory, methodology and design. Springer, London, pp 49-72

11. Mynatt ED, Back M, Want R, Baer M, Ellis JB (1998) Designing audio aura. In: CHI'98: proceedings of the SIGCHI conference on human factors in computing systems, pp 566-573

12. Mauney BS, Walker BN (2004) Creating functional and livable soundscapes for peripheral monitoring of dynamic data. In:
ICAD'04: proceedings of he 10th international conference on auditory display

13. Pashler HE (1998) The psychology of attention. MIT Press, Cambridge

14. Treisman AM (1964) Verbal cues, language, and meaning in selective attention. Am J Psychol 77:206-219

15. Moray N (1959) Attention in dichotic listening: affective cues and the influence of instructions. Q J Exp Psychol 11:56-60

16. Kahneman D (1973) Attention and effort. Prentice-Hall, Englewood Cliffs

17. Wickens CD, Hollands JG (2000) Engineering psychology and human performance. Prentice-Hall, Englewood Cliffs

18. Bakker S, van den Hoven E, Eggen B (2010) Design for the periphery. In: Eurohaptics 2010: proceedings of the special symposium haptic and audio-visual stimuli, pp 71-80

19. Sleeswijk Visser F, Stappers JP, van der Lugt R, Sanders EBN (2005) Contextmapping: experiences from practice. CoDesign 1:119-149

20. Gaver B, Dunne T, Pacenti E (1999) Design: cultural probes. ACM Interact 6:21-29

21. Liamputtong P, Ezzy D (2005) Qualitative research methods. Oxford University Press, Melbourne

22. Hsieh H, Shannon SE (2005) Three approaches to qualitative content analysis. Qual Health Res 15:1277-1288

23. Siegel S, Castellan NJ (1988) Nonparametric statistics for the behavioral sciences. McGraw-Hill, Boston

24. Landis JR, Koch GG (1977) The measurement of observer agreement for categorical data. Biometrics 33:159-174

25. James W (1890) The principles of psychology. Dover, New York 